\title{
First single-incision type 7 total laparoscopic hysterectomy, adnexectomy, and appendectomy
}

\author{
Katherine A. O'Hanlan
}

Received: 18 May 2009 / Accepted: 9 July 2009 / Published online: 24 July 2009

(C) Springer-Verlag 2009

\begin{abstract}
We report two cases of use of a single umbilical skin incision for a type 7 total laparoscopic hysterectomy, bilateral salpingo-oophorectomy, and appendectomy. This study is a retrospective chart review and discussion of two patients who underwent a total laparoscopic hysterectomy, bilateral salpingo-oophorectomy, and appendectomy (Canadian Task Force Level III). Both patients underwent a type 7 total laparoscopic hysterectomy for benign indications in July 2007 and sustained no complications. The evidence from these two cases suggests that advanced laparoscopic procedures are possible using a single skin incision for multiple ports. Technological advances, including those in port structure, are needed to enable surgeons to employ strategies that effectively enhance instrument coordination and suturing. Benefits to the patient need to be documented prospectively before this procedure can be recommended widely.
\end{abstract}

Keywords Total laparoscopic hysterectomy .

Single-incision hysterectomy $\cdot$ SILS $\cdot$ SPA $\cdot$ TLH

\section{Introduction}

Type 7 total laparoscopic hysterectomy (TLH), bilateral salpingo-oophorectomy (BSO) is a hysterectomy in which every step is performed via the laparoscopic ports,

Katherine A. O'Hanlan is a consultant and speaker for Covidien and Novare Surgical Instruments.

K. A. O’Hanlan $(\bowtie)$

Laparoscopic Institute for Gynecologic Oncology,

4370 Alpine Road Suite 104,

Portola Valley, CA 94028, USA

e-mail: ohanlan@AOL.com including suturing of the vaginal cuff [1]. TLH/BSO and incidental appendectomy are now performed routinely at community and university hospitals worldwide for both malignant and benign indications. The standard of four $5-\mathrm{mm}$ ports in a diamond configuration has been reported to be effective for both pelvic and abdominal procedures [2]; however, there has been much interest in singleincision laparoscopic surgery. Pelosi and colleagues used a single umbilical port to facilitate a type 3 laparoscopicassisted vaginal hysterectomy/BSO, a supracervical hysterectomy/BSO, and an appendectomy [3-5]. These authors summarized: "The results suggest single-puncture (minilaparoscopy) operative endoscopy as the ultimate goal in the progression of minimally invasive surgery" [5]. However, widespread interest lagged until recent technological advances in laparoscopic equipment allowed for easier applications of standard laparoscopic surgical techniques through very closely placed ports. King (Stephanie A. King, personal communication) with Curcillo and other colleagues, has been performing single-incision BSO and other procedures since 2007 [6, 7].

\section{Patients and methods}

In July 2007, two patients were consented for TLH/BSO/ appendectomy by a surgeon experienced in type 7 TLH, with the understanding that as much of the procedure as possible would be accomplished using one larger umbilical incision for three ports; however, as many as three additional ports would be added to facilitate the procedure if progress in the surgery became slowed due to port location difficulties. The standard instruments and techniques of type 7 TLH/BSO/appendectomy used by this surgeon in over 800 cases beforehand are described 
elsewhere and were employed in standard fashion in these two cases $[2,8]$. The procedure started by injecting the skin and fascia with $10 \mathrm{~cm}^{3}$ bupivacaine $0.25 \%$ and making a $3-\mathrm{cm}$ incision through the umbilicus, extending $1 \mathrm{~cm}$ above it and $1 \mathrm{~cm}$ below it (Fig. 1). The skin edges of the umbilicus were elevated with towel clips at the apical scar and the first port was bluntly inserted directly into the uninsufflated abdomen [9], followed by the next two after insufflation with carbon dioxide to $12 \mathrm{mmHg}$ pressure. For both cases, no other ports were utilized and no other sites were employed other than the three 5-mm ports entering through the umbilicus. The surgeon stood on the patient's right side, and the assistant stood on the patient's left. Through the three 5-mm ports, surgeons used a $5-\mathrm{mm} 30^{\circ}$ lens (Stryker, San Jose, CA, USA), a 5-mm Ligasure (Covidien, Boulder, CO, USA), and a bullet tip RealHand grasper (Novare Surgical Devices, San Jose, CA, USA). After identification of the ureter, the infundibulopelvic ligament was cauterized and incised. The broad and round ligaments were incised. Then the uterus was elevated on a V-Care Uterine manipulator (ConMed, Utica, NY, USA) so that the bladder flap could be opened to expose the glistening white anterior cervical fascia. The uterine arteries were coagulated multiple times because the tissue was on traction upward. Then the uterine arteries were incised, exposing the cardinal and uterosacral ligament fibers. These were incised along with the anterior pubocervical fascia in a coagulating fashion, gradually entering the vagina directly on the manipulator cervical cup. After incising $360^{\circ}$, the uterus and ovaries were delivered through the vagina. Pneumoperitoneum was maintained by placing a glove with two four-by-four gauze sponges in the vagina. At this point, the appendix was elevated with the grasper to allow the mesoappendix to be coagulated and incised down to the

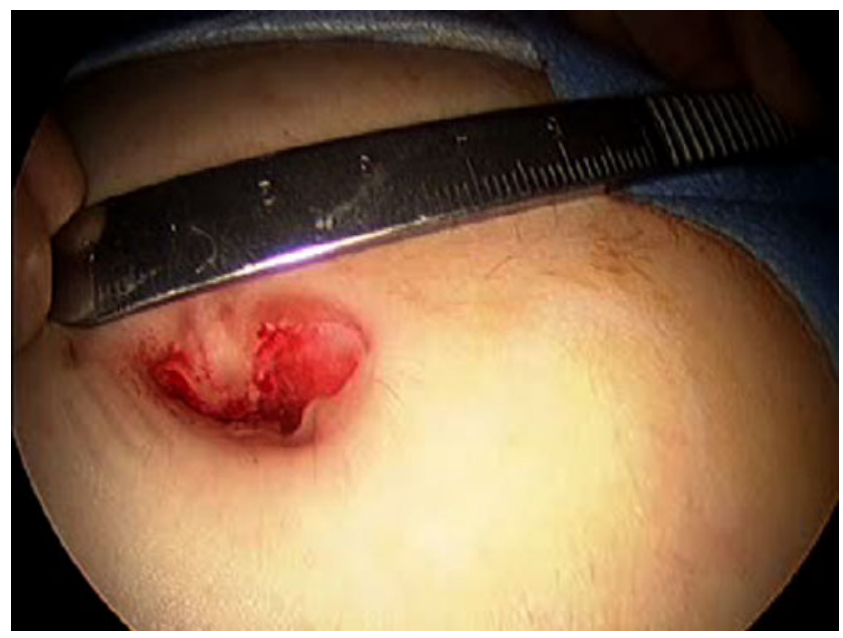

Fig. 1 The post-procedure incision is $3 \mathrm{~cm}$. Note that the patient has a ring piercing above the umbilicus. This was not injured by this incision peritoneal window at the base. An EJ-10G Endloop 0 vicryl suture (Ethicon Endosurgery, Cincinnati, OH, USA) with a pre-tied lasso knot and was strictured around the base with the preloaded plastic pusher. Then the appendix was cauterized and cut away with the LigaSure bipolar device and pulled out through the vagina on a ring forceps. After ascertaining hemostasis, the vagina was closed using JK-10G Endoknot 0 vicryl suture (Ethicon Endosurgery) on a straight ST-3 needle. Using a 5-mm Semb toothed, cupped biopsy RealHand device (Novare Surgical Devices) as an articulating grasper for the vagina, three "figure of $\mathrm{N}$ " spiral sutures were applied laparoscopically. The incision was closed in a subcuticular fashion using 4-0 vicryl and Dermabond (Ethicon Endosurgery). Patients were discharged the following morning.

\section{Results}

Case 1 A 39-year-old nulligravid woman with body mass index of $28 \mathrm{~kg} / \mathrm{m}^{2}$ complained of chronic pelvic pain and disabling dysmenorrhea. Preoperative ultrasound revealed normal pelvic structures. A serum Ca125 was 34 . Using a single 3-cm trans-umbilical skin incision, three 5-mm Apple ports were inserted via three different fascial locations, in an equilateral triangular distribution (Fig. 2). A $5-\mathrm{mm} 30^{\circ}$ Stryker laparoscope, a Novare RealHand grasper, and a Covidien 5-mm Ligasure were employed through the three umbilical ports. The total procedure time was $58 \mathrm{~min}$, with an estimated blood loss of $5 \mathrm{~cm}^{3}$ based on the aspirated fluid volume in the cul-de-sac at the end of the procedure (Fig. 3). The uterus weighed $113 \mathrm{~g}$ and had benign endometriosis on the surface. The patient was discharged within the 23-h admission parameters, and recovered without complication.

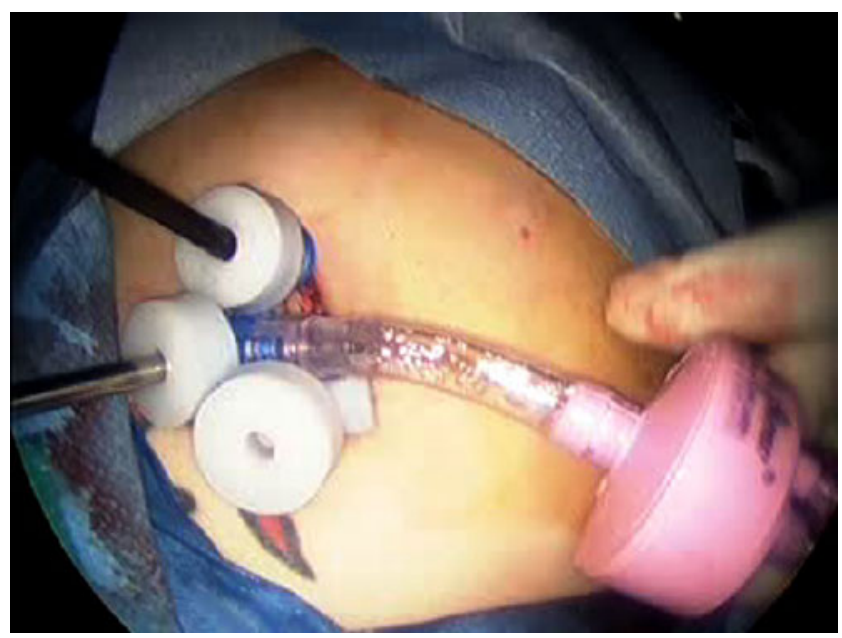

Fig. 2 The Insufflow device heats and humidifies carbon dioxide going through one of the three low-profile ports 


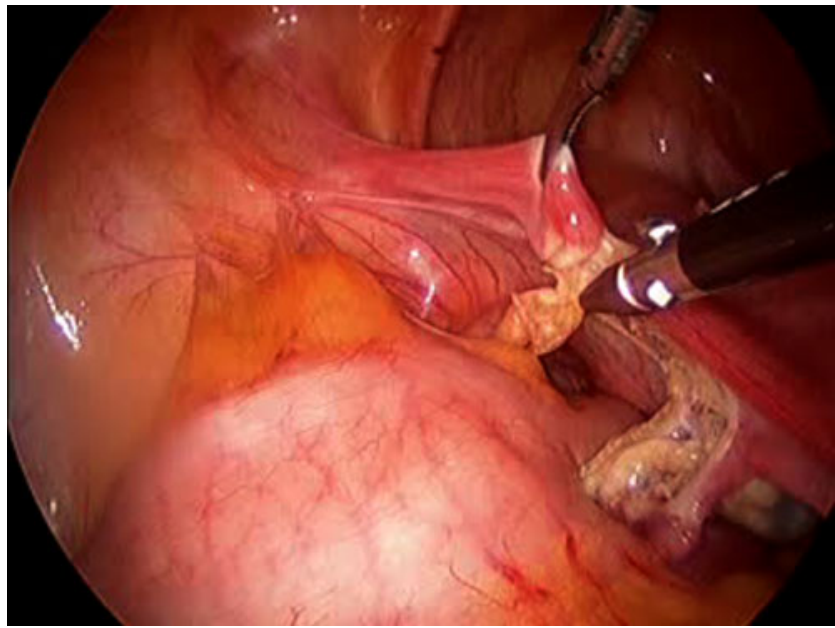

Fig. 3 The RealHand grasper appears to be coming from a port remote from the $30^{\circ}$ scope and the Ligasure due to its easy intracavitary flexibility. It is grasping the left round ligament for the Ligasure $\mathrm{V}$ to seal. Notice the sealed edges of the cut ovarian vessels (bottom) in this picture in which the surgical focus is on the right side of the picture. The eccentric view facilitates surgical manipulation

Case 2 A 48-year-old nulligravid women with a body mass index of $43 \mathrm{~kg} / \mathrm{m}^{2}$ had large fibroids, with menorrhagia, pelvic pressure, and urinary frequency. Exam suggested that the uterus was about 16 weeks in gestational size, and ultrasound demonstrated uterine fibroids, with normal ovaries. The TLH/BSO/appendectomy took 176 min, with $200 \mathrm{~cm}^{3}$ of measured blood loss. The uterus weighed $535 \mathrm{~g}$ and contained only benign leiomyomata uteri. The patient was discharged home the following day and recovered without complication.

\section{Discussion}

Single-incision laparoscopic surgery may theoretically result in less pain for the patient, but prospective data are needed to justify the extra challenges to the surgeons. Multiple challenges to success of this technique exist.

Patient selection for the first cases should include only those who could be completed as a standard four-port TLH. These two patients were comparable to those in the series of 830 patients published by the author in 2006 . Their mean age was 50 years, parity was 1.3 , body mass index was $28 \mathrm{~kg} / \mathrm{m}^{2}$, duration of surgery was $132 \mathrm{~min}$, estimated blood loss was $130 \mathrm{~cm}^{3}$, uterine weight was $160 \mathrm{~g}$, hospital stay was 1 day, and serious complication rate was $4.7 \%$ in series (none in these two patients) were similar [2].

The procedure is technically much more difficult to perform due to motion limitations of the laparoscopic equipment at the port sites. Surgeons in both cases above noted that their hands on the instrument and scope handles

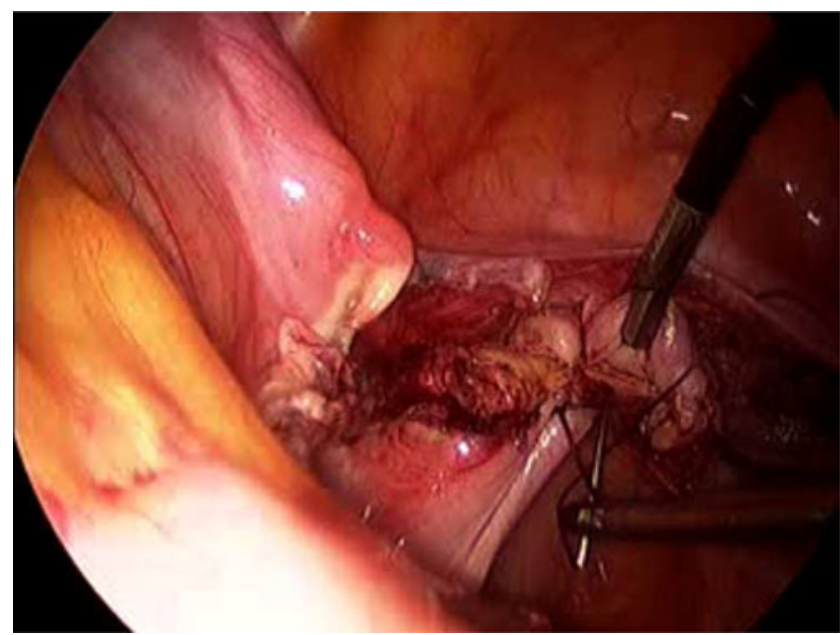

Fig. 4 The RealHand grasper holding the anterior vaginal wall for closure with three "figure of N's" with the JK10 suture. Note the eccentric view

frequently competed for optimal functional locations during the surgery due to the proximity of the three ports in the umbilical incision. They devised a collaboration that allowed the visual focus of the surgery on the monitor to be at the side of the large picture, instead of in the middle of the monitor picture, allowing the operative hands slightly more room to operate.

Fader and colleagues have performed 13 single-incision procedures in 2009, using robotic instrumentation for some [10]. At the time of the above two surgeries, there were no reports of type 7 total laparoscopic hysterectomy. However, since July 2007, newer technologies that have enabled consideration for these advanced and difficult procedures being performed through one opening with the three small ports bear mention. Frequent experimentation with rotations of the $30^{\circ}$ Stryker scope (Stryker) allowed more room for

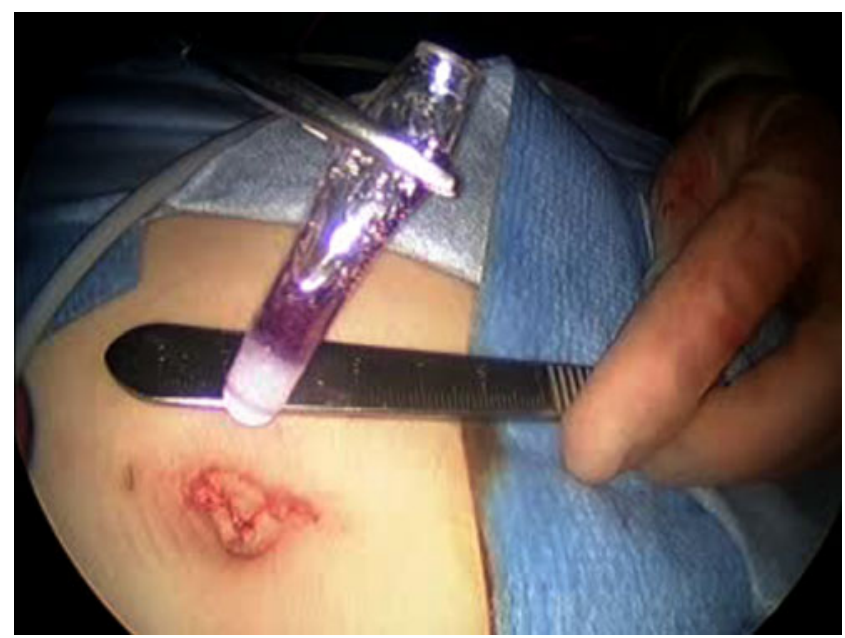

Fig. 5 Application of Dermabond to the incision closed by subcuticular 4-0 vicryl and sealed by Dermabond. Note that the umbilical piercing is intact 
manual operative manipulation of the grasping and bipolarcutting instruments. The optics of this scope system enables a broad field of view with high-definition resolution. Stryker now offers a flexible fiber optic scope that articulates within the abdominal cavity, which may make future cases easier to perform. The rotational flexibility of the RealHand device (Novare Surgical Devices) made the procedure possible due to the $360^{\circ}$ round articulation of the graspers to greater than $90^{\circ}$ angle of deflection [11]. Use of Ligasure $\mathrm{V}$ bipolar $5 \mathrm{~mm}$ device (Covidien) gave proven vessel sealing accuracy in this case where anatomy and limited angulation were challenging [12]. This device now is available with both monopolar and bipolar functionalities, which may make future single-incision cases easier. Finally, new ports have been designed that allow three instruments to have much more freedom of motion, likely reducing the competition for space by the surgeon's and assistant's hands. Lastly, the vagina in both cases was closed using three "figure of N's" of 0-vicryl on a straight 1-in. ST-3 needle with a disposable knot puller, making closure of the vagina laparoscopically much easier to accomplish (Fig. 4).

\section{Conclusions}

New technology has made type 7 total laparoscopic hysterectomy, bilateral salpingo-oophorectomy, and appendectomy possible through a single umbilical skin incision (Fig. 5), but these procedures require prospective randomized trials to confirm any real benefit to the patient as they are challenging to perform. To our knowledge, this is the first reported type 7 total laparoscopic hysterectomy, adnexectomy, and appendectomy. Devel- oping technologies will make this approach more feasible in the future.

\section{References}

1. Nezhat C, Nezhat F, Admon D, Nezhat AA (1995) Proposed classification of hysterectomies involving laparoscopy. J Am Assoc Gynecol Laparosc 2(4):427-429

2. O'Hanlan KA, Dibble SL, Garnier AC, Reuland ML (2007) Total laparoscopic hysterectomy: technique and complications of 830 cases. JSLS 11(3):45-53

3. Pelosi MA, Pelosi MA 3rd (1991) Laparoscopic hysterectomy with bilateral salpingo-oophorectomy using a single umbilical puncture. N J Med 88(10):721-726

4. Pelosi MA, Pelosi MA 3rd (1992) Laparoscopic supracervical hysterectomy using a single-umbilical puncture (mini-laparoscopy). J Reprod Med 37(9):777-784

5. Pelosi MA, Pelosi MA 3rd (1992) Laparoscopic appendectomy using a single umbilical puncture (minilaparoscopy). J Reprod Med 37(7):588-594

6. Castellucci SA, Curcillo PG, Ginsberg PC, Saba SC, Jaffe JS, Harmon JD (2008) Single port access adrenalectomy. J Endourol 22(8):1573-1576

7. Podolsky ER, Rottman SJ, Curcillo PG 2nd (2009) Single port access (SPA) gastrostomy tube in patients unable to receive percutaneous endoscopic gastrostomy placement. Surg Endosc 23(5):1142-1145

8. O'Hanlan KA, Fisher DT, O'Holleran MS (2007) 257 incidental appendectomies during total laparoscopic hysterectomy. JSLS 11 (4):428-431

9. Jacobson MT, Helmy M, Smith KS, Nezhat CH, Nezhat F, Nezhat CR (2000) Transumbilical direct trocar entry for operative videolaparoscopy. Obstet Gynecol 95(4 Suppl 1):S33

10. Fader AN, Escobar PF (2009) Laparoendoscopic single-site surgery (LESS) in gynecologic oncology: technique and initial report. Gynecol Oncol 114(2):157-161

11. Rettenmaier MA, Lopez K, Graham CL et al (2009) Realhand high dexterity instruments for the treatment of stage I uterine malignancy. JSLS 13(1):27-31

12. Lamberton GR, Hsi RS, Jin DH, Lindler TU, Jellison FC, Baldwin DD (2008) Prospective comparison of four laparoscopic vessel ligation devices. J Endourol 22(10):2307-2312 